\title{
FBST FOR COVARIANCE STRUCTURES OF GENERALIZED GOMPERTZ MODELS
}

\author{
Viviane Teles de Lucca Maranhão*,**, \\ Marcelo de Souza Lauretto ${ }^{+}$, Julio Michael Stern* \\ IME-USP* and EACH-USP ${ }^{+}$, University of São Paulo \\ vivianedime.usp.br**
}

\begin{abstract}
.
The Gompertz distribution is commonly used in biology for modeling fatigue and mortality. This paper studies a class of models proposed by Adham and Walker, featuring a Gompertz type distribution where the dependence structure is modeled by a lognormal distribution, and develops a new multivariate formulation that facilitates several numerical and computational aspects. This paper also implements the FBST, the Full Bayesian Significance Test for pertinent sharp (precise) hypotheses on the lognormal covariance structure. The FBST's $e$-value, ev $(H)$, gives the epistemic value of hypothesis, $H$, or the value of evidence in the observed in support of $H$.
\end{abstract}

Keywords: Full Bayesian Significance Test, Evidence, Multivariate Gompertz models

\section{INTRODUCTION}

This paper presents a framework for testing covariance structures in biological survival data. Gavrilov $(1991,2001)$ and Stern (2008) motivate the use of Gompertz type distributions for survival data of biological organisms. Section 2 presents Adham and Walker (2001) characterization of the univariate Gompertz Distribution as a Gamma mixing stochastic process, and the Gompertz type distribution obtained by replacing the Gamma mixing distribution by a Log-Normal approximation. Section 3 presents the multivariate case. Section 4 presents the formulation of the FBST for sharp hypotheses about the covariance structure in these models. Section 5 presents some details concerning efficient numerical optimization and integration procedures. Section 6 and 7 present some experimental results and our final remarks.

\section{THE UNIVARIATE LOG-NORMAL GOMPERTZ DISTRIBUTION}

This section presents Adham and Walker (2001) characterization of the (reparameterized) univariate Gompertz Distribution as a Gamma mixing stochastic process. Furthermore, Adham and Walker (2001) suggest the use of a Log-Normal approximation for the Gamma mixing distribution that greatly simplifies both numerical computations and multivariate extensions of the univariate model. Section 7 of Pereira and Stern (2008) describe similar uses of Log-Normal approximations to the Gamma distribution, see also Aitchison and Shen (1980). In many examples of the authors consulting practice these approximations proved to be a powerful modeling tool, leading to efficient computa- 
tional procedures.

A non-negative random variable $t$ follows a Univariate Gompertz distribution with parameters $a$ and $c$, if its distribution function is given by:

$$
f(t \mid a, c)=f(t)=a c \exp (a t) \exp (-c(\exp (a t)-1)) .
$$

Adham and Walker (2001) show that we can rewrite the previous density with parameters $a>0$ e $c>0$ as a product of mixtures using the Gamma distribution, $\Gamma(2, c)$, as follow:

$$
f(t \mid u)=a u^{-1} \exp (a t) \mathbf{I}[u>\exp (a t)-1] \text { and } f(u)=\Gamma(2, c)=c^{2} u \exp (-c u) .
$$

In their work, Adham and Walker (2001) introduce the GOLN distribution, an alternative to the Gompertz, which uses the representation of mixtures with a log-normal distribution $L N\left(\mu, \sigma^{2}\right)$ whose parameters are determined by the minimum KullbackLeibler distance for the gamma distribution $\Gamma(2, c)$. The final formula has Gaussian core and is given by:

$$
\begin{aligned}
& f(t \mid u)=a \exp (a t) \exp (-u) \mathbf{I}[u>\log (\exp (a t)-1)] \text { and } \\
& u \sim N\left(\mu, \sigma^{2}\right), \mu=E(\log (x)), \sigma^{2}=E\left\{(\log (x))^{2}\right\}-\mu^{2}, x \sim \Gamma(2, c) .
\end{aligned}
$$

Lemma We can write the GOLN distribution as follows:

$$
f(t)=a \exp \left(a t-\mu+\frac{\sigma^{2}}{2}\right)\left[1-\Phi\left(\frac{\log (\exp (a t)-1)-\mu+\sigma^{2}}{\sigma}\right)\right],
$$

where $\Phi(\cdot)$ is the cumulative probability function of standard normal distribution.

Proof:

Using the law of total probability for $f(t)$ from its representation of mixtures, we have:

$$
f(t)=\int_{\Omega} f(t \mid u) f(u) d u=a \exp (a t) \int_{\log (\exp (a t)-1)}^{\infty} \frac{1}{\sigma \sqrt{2 \pi}} \exp \left(-u-\frac{(u-\mu)^{2}}{2 \sigma^{2}}\right) d u .
$$

Adding and subtracting $\mu$ of the integral's exponent, we have

$$
f(t)=a \exp (a t-\mu) \int_{\log (\exp (a t)-1)}^{\infty} \frac{1}{\sigma \sqrt{2 \pi}} \exp \left(-(u-\mu)-\frac{(u-\mu)^{2}}{2 \sigma^{2}}\right) d u .
$$

Using the change of variables $v=u-\mu$ and $d v=d u$

$$
f(t)=a \exp (a t-\mu) \int_{\log (\exp (a t)-1)-\mu}^{\infty} \frac{1}{\sigma \sqrt{2 \pi}} \exp \left(-v-\frac{v^{2}}{2 \sigma^{2}}\right) d v .
$$

Using the change of variables $y=v+\alpha$, it is possible to rewrite the integral's exponent as

$$
-v-\frac{v^{2}}{2 \sigma^{2}}=\frac{-y^{2}-2 y\left(\sigma^{2}+\alpha\right)-\alpha\left(2 \sigma^{2}+\alpha\right)}{2 \sigma^{2}} .
$$


Considering the last equality as a quadratic equation in $\mathrm{y}$, we can eliminate the linear term by taking $\alpha=-\sigma^{2}$ and get

$$
-v-\frac{v^{2}}{2 \sigma^{2}}=\frac{-y^{2}}{2 \sigma^{2}}+\frac{\sigma^{2}}{2}
$$

Using one more change of variables, $y=v+\sigma^{2}$ and $d y=d v$, we can re-write the integral as

$$
f(t)=a \exp \left(a t-\mu+\frac{\sigma^{2}}{2}\right) \int_{\log (\exp (a t)-1)-\mu+\sigma^{2}}^{\infty} \frac{1}{\sigma \sqrt{2 \pi}} \exp \left(\frac{-y^{2}}{2 \sigma^{2}}\right) d y .
$$

After another change of variables, $w=\frac{y}{\sigma}$ and $d w=\frac{d y}{\sigma}$, we get

$$
f(t)=a \exp \left(a t-\mu+\frac{\sigma^{2}}{2}\right) \int_{\frac{\log (\exp (a t)-1)-\mu+\sigma^{2}}{\sigma}}^{\infty} \frac{1}{\sqrt{2 \pi}} \exp \left(\frac{-w^{2}}{2}\right) d w .
$$

Hence, we can see that the integrand is the probability density of the random variable $w$ which follows standard normal distribution. In this case, it is worth noticing that $P(A \leq w \leq B)=\Phi(B)-\Phi(A)$. Hence, remembering that $\Phi(\infty)=1$, we have

$$
f(t)=a \exp \left(a t-\mu+\frac{\sigma^{2}}{2}\right)\left[1-\Phi\left(\frac{\log (\exp (a t)-1)-\mu+\sigma^{2}}{\sigma}\right)\right] \text { Q.E.D. }
$$

Lemma In order to get a good GOLN approximation to the Gompertz distribution with parameters $a>0$ and $c>0$, we can choose the parameters of the normal distribution as follows:

$$
\mu=1-\gamma-\log (c) \text { and } \sigma^{2}=\pi^{2} / 6-1,
$$

where $\gamma \approx 0.5572156$ is the Euler-Mascheroni constant.

Proof:

$$
\begin{aligned}
\mu & =E_{\Gamma(2, c)}[\log (x)]=\int_{0}^{\infty} c^{2} x \exp (-c x) \log (x) d x=1-\gamma-\log (c) \\
\sigma^{2} & =E_{\Gamma(2, c)}\left[\log (X)^{2}\right]-\mu^{2}=\int_{0}^{\infty} c^{2} x \exp (-c x) \log (x)^{2} d x-(1-\gamma-\log (c))^{2} \\
& =\left[\gamma^{2}-2 \gamma+\frac{\pi^{2}}{6}-2 \log (c)-2 \gamma \log (c)+\log (c)^{2}\right]- \\
& -\left[-1+2 \gamma+2 \log (c)-\gamma^{2}-2 \gamma \log (c)-\log (c)^{2}\right] \\
& =\frac{\pi^{2}}{6}-1 \text { Q.E.D. }
\end{aligned}
$$




\section{MULTIVARIATE GOMPERTZ LOG-NORMAL DISTRIBUTION}

Adham and Walker (2001) present the $p$-dimensional GOLN distribution for a random variable $T=\left(t_{1}, \cdots, t_{p}\right)$ with parameters $A=\left(a_{1}, \cdots, a_{p}\right)$ and $C=\left(c_{1}, \cdots, c_{p}\right)$. This is an extension of one-dimensional representation of mixtures, based on $U=\left(u_{1}, \cdots, u_{p}\right)$, a multivariate normal distribution. The construction of the multivariate GOLN is as follows:

$$
\begin{aligned}
f(T \mid U) & =\prod_{j=1}^{p} f\left(t_{j} \mid u_{j}\right)=\prod_{j=1}^{p} a_{j} \exp \left(a_{j} t_{j}-u_{j}\right) \mathbf{I}\left[u_{j}>\log \left(\exp \left(a_{j} t_{j}\right)-1\right)\right] \\
& =K \exp \left(A^{\prime} T-\mathbf{1}^{\prime} U\right) \mathbf{I}[B]
\end{aligned}
$$

where

$$
\begin{gathered}
K=\prod_{j=1}^{p} a_{j}, \mathbf{1}=\left\{x \in \mathbb{R}^{p} \mid x_{j}=1\right\}, \\
B=\left\{u \in \mathbb{R}^{p} \mid u_{j}>\log \left(\exp \left(a_{j} t_{j}\right)-1\right)\right\}, \text { and } \\
U \sim M V N(M, \Sigma), M=\left(\mu_{1}, \cdots, \mu_{p}\right)^{\prime}, \Sigma=\left[\sigma_{i j}\right]_{p \times p}, \sigma_{i i}=\operatorname{var}\left(u_{i}\right), \sigma_{i j}=\operatorname{cov}\left(u_{i}, u_{j}\right) .
\end{gathered}
$$

Lemma We can write the $p$-dimensional GOLN distribution with parameters $A, M$ and $\Sigma, a_{i}, \mu_{i}>0, i=1 \cdots, p$ of a random variable $T$ as follows:

$$
\begin{aligned}
& f(T)=\exp \left(\mathbf{1}^{\prime} \log (A)+A^{\prime} T-\mathbf{1}^{\prime} M+\frac{1}{2} \mathbf{1}^{\prime} \Sigma \mathbf{1}+\log \left(\Phi\left(B^{\prime \prime}\right)\right)\right) \text { where } \\
& B^{\prime \prime}=\left\{u \in \mathbb{R}^{p} \mid u_{j}>\log \left(\exp \left(a_{j} t_{j}\right)-1\right)-\mu_{j}+\sum_{k=1}^{p} \sigma_{j k}\right\},
\end{aligned}
$$

$\Phi(X)$ is the "cumulative probability function" of a p-variate normal distribution, $\operatorname{MVN}(0, \Sigma)$, in the range $[X, \infty]$. As it is usual for scalar functions taking vector arguments, the $\log (X)$ operator is applied element by element on vector $X$.

Proof: The demonstration is similar to the one-dimensional case. We start from $f(T)$ represented as a mixture and use the law of total probability

$$
f(T)=\int_{\Omega} f(t \mid u) f(u) d u=\int_{B} K \frac{\exp \left(A^{\prime} T-\mathbf{1}^{\prime} U\right)}{\sqrt{|2 \pi \Sigma|}} \exp \left(-\frac{1}{2}(U-M)^{\prime} \Sigma^{-1}(U-M)\right) d U .
$$

Adding and subtracting $\mathbf{1}^{\prime} M$ to the integral's exponent and taking the change of variables $V=U-M$ and $d V=d U$, we have

$$
\begin{aligned}
& f(T)=\prod_{A} \exp \left(A^{\prime} T-\mathbf{1}^{\prime} M\right) \int_{B^{\prime}} \frac{\exp \left(-\mathbf{1}^{\prime} V-\frac{1}{2} V^{\prime} \Sigma^{-1} V\right)}{\sqrt{|2 \pi \Sigma|}} d V \text { with } \\
& B^{\prime}=\left\{u \in \mathbb{R}^{p} \mid u>\log \left(\exp \left(a_{j} t_{j}\right)-1\right)-\mu_{j}\right\} .
\end{aligned}
$$


Using the change of variables $V=Y+\Lambda$, and remembering that matrix $\Sigma^{-1}$ is symmetric, it is possible to rewrite the integral's exponent as

$$
-\frac{1}{2} V^{\prime} \Sigma^{-1} V=-\frac{1}{2} Y^{\prime} \Sigma^{-1} Y-\left(\Sigma^{-1} \Lambda+\mathbf{1}\right)^{\prime} Y+\left(-\frac{1}{2} \Lambda-\mathbf{1}\right)^{\prime} \Lambda .
$$

Considering the last equality as a quadratic equation in $Y$, we can eliminate the linear term by taking $\Lambda=-\Sigma^{2}$ and get

$$
-\frac{1}{2} V^{\prime} \Sigma^{-1} V=-\frac{1}{2} Y^{\prime} \Sigma^{-1} Y+\frac{1}{2} \mathbf{1}^{\prime} \Sigma \mathbf{1}
$$

Using one more change of variables, $Y=V+\Sigma 1$ and $d Y=d V$, we can re-write the integral as

$$
\begin{aligned}
& f(T)=K \exp \left(A^{\prime} T-\mathbf{1}^{\prime} M+\frac{1}{2} \mathbf{1}^{\prime} \Sigma \mathbf{1}\right) \int_{B^{\prime \prime}} \frac{\exp \left(-\frac{1}{2} Y^{\prime} \Sigma^{-1} Y\right)}{\sqrt{|2 \pi \Sigma|}} d Y \text { with } \\
& B^{\prime \prime}=\left\{u \in \mathbb{R}^{p} \mid u_{j}>\log \left(\exp \left(a_{j} t_{j}\right)-1\right)-\mu_{j}+\sum_{k=1}^{p} \sigma_{j k}\right\},
\end{aligned}
$$

where the integrand is the centered multivariate Normal distribution, $N(0, \Sigma)$. Hence, we have

$$
f(T)=K \exp \left(A^{\prime} T-\mathbf{1}^{\prime} M+\frac{1}{2} \mathbf{1}^{\prime} \Sigma \mathbf{1}\right) \Phi\left(B^{\prime \prime}\right) .
$$

Moving everything to the exponential, we finally obtain

$$
f(T)=\exp \left(\mathbf{1}^{\prime} \log (A)+A^{\prime} T-\mathbf{1}^{\prime} M+\frac{1}{2} \mathbf{1}^{\prime} \Sigma \mathbf{1}+\log \left(\Phi\left(B^{\prime \prime}\right)\right)\right), \text { Q.E.D. }
$$

\section{FBST FOR GOLN COVARINCE STRUCTURES}

The FBST and its significance measure, the $e$-value - ev $(H)$, were specially designed to handle sharp or precise hypotheses. Further details, demonstration of theoretical properties, comparison with other statistical tests for sharp hypotheses, and an extensive list of references, see Borges and Stern (2007) and Pereira et al. (2008).

\section{Likelihood and Posteriori distribution}

The likelihood function of multivariate GOLN, with sample size $n$ where the $i$-th observation is denoted $T^{(i)}$, is given by:

$$
\begin{aligned}
L(A, M, \Sigma \mid T) & =\prod_{i=1}^{n} \exp \left(\mathbf{1}^{\prime} \log (A)+A^{\prime} T^{(i)}-\mathbf{1}^{\prime} M+\frac{1}{2} \mathbf{1}^{\prime} \Sigma \mathbf{1}+\log \left(\Phi\left(B^{\prime \prime(i)}\right)\right)\right) \\
& =\exp \left(\sum_{i=1}^{n}\left(\mathbf{1}^{\prime} \log (A)+A^{\prime} T^{(i)}-\mathbf{1}^{\prime} M+\frac{1}{2} \mathbf{1}^{\prime} \Sigma \mathbf{1}+\log \left(\Phi\left(B^{\prime \prime(i)}\right)\right)\right)\right) .
\end{aligned}
$$


Assuming the following independent priors for the parameters,

$$
\begin{aligned}
\pi\left(a_{j}\right) & \propto 1 / a_{j} \\
\pi(C) & \sim \operatorname{MVLN}(0, \Omega) \\
\pi\left(\Sigma^{-1}\right) & \sim \operatorname{Wishart}\left(p,(p R)^{-1}\right) \quad \text { with } \\
\Omega & =\operatorname{diag}\left(\tau_{j j}^{2}\right), \quad \tau_{j j}^{2}=E=100 ; j=1, \ldots, p \\
R & =\operatorname{diag}\left(r_{j j}\right), \quad r_{j j}=\varepsilon=0.1 ; j=1, \ldots, p
\end{aligned},
$$

and the previously established relationship on $\mu$ and $c$ for the multivariate case,

$$
M=1-\gamma-\log (C), \quad \gamma \approx 0.5572156,
$$

we can write the GOLN's model posterior as follows:

$$
f(A, M, \Sigma \mid T) \propto \pi(A, M, \Sigma) l(A, M, \Sigma \mid T)
$$

with

$$
\pi(A, M, \Sigma)=\left(\prod_{j=1}^{p} \pi\left(a_{j}\right)\right) \pi(M) \pi\left(\Sigma^{-1}\right) .
$$

Hence, the log-posterior is:

$$
l p(A, M, \Sigma \mid t)=l \pi(A)+l \pi(M)+l \pi\left(\Sigma^{-1}\right)+l L,
$$

with

$$
\begin{aligned}
l \pi(A) & =l \pi(A, \mid T)=-\sum_{j=1}^{p} \log \left(a_{j}\right) \\
l \pi(M) & =l \pi(C \mid \Omega, T) \propto-\frac{1}{2} \sum_{j=1}^{p}\left(\frac{\log \left(\mu_{j}\right)^{2}}{E}+\log \left(\mu_{j}\right)\right) ; \\
l \pi\left(\Sigma^{-1}\right) & =l \pi\left(\Sigma^{-1} \mid T\right) \propto-\frac{1}{2}\left(\log \left(\left|\Sigma^{-1}\right|\right)+p \varepsilon \operatorname{tr}\left(\Sigma^{-1}\right)\right) ; \\
l L & =l L(A, M, \Sigma \mid T)= \\
& =n\left[\mathbf{1}^{\prime}\left(\log (A)-M+\frac{1}{2} \Sigma 1\right)\right]+A^{\prime}\left(\sum_{i=1}^{n} T^{(i)}\right)+\sum_{i=1}^{n} \log \left(\Phi\left(B^{\prime \prime(i)}\right)\right) .
\end{aligned}
$$

\section{Null Hypothesis}

We will consider hypotheses on the structure of the covariance matrix $\Sigma$ of GOLN models, using exactly the same notation defined in Lauretto et al. (2003). Hence, $\Sigma=$ 
$\Sigma(\gamma)=\sum_{h=1}^{m} \gamma_{h} G^{h}, m=(1 / 2) p(p+1)$ where the matrices $G^{h}$ form a basis for the space of $p \times p$ symmetric matrices. Including the calibration or proportionality parameter $\delta$ into the parameter space, we have:

$$
\begin{aligned}
\Theta & =\left\{\theta=\left[A^{\prime}, M^{\prime}, \gamma^{\prime}, \delta\right]^{\prime} \in \mathbb{R}^{4+4+10+1}, \Sigma(\gamma)>0\right\} \\
\Theta_{0} & =\{\theta \in \Theta \mid h(\theta)=0\}
\end{aligned}
$$

where $h(\theta)$ is a structural hypothesis with parameters $\gamma$ and $\delta$.

\section{NUMERICAL OPTIMIZATION AND INTEGRATION}

As in Lauretto et al. (2003), we adopt the following objective function to be maximized:

$$
f(A, \mu, \Sigma \mid T)=l p-\rho p \text { frob2 }(\Sigma-S), \text { where frob2 }(M)=\sum_{i=1}^{p} \sum_{j=1}^{p}\left(M_{i j}\right)^{2} .
$$

$\rho$ is a regularization factor that tends to zero as the optimization proceeds, and $S$ is the empirical covariance, for details, see Lauretto et al. (2003).

The gradients of the objective function are:

$$
\begin{aligned}
& \frac{\partial f(A, M, \Sigma \mid T)}{\partial a_{j}}=\frac{n-1}{a_{j}}+\sum_{i=1}^{n}\left[t_{j}^{(i)}\right]+\sum_{i=1}^{n} \frac{-t_{j}^{(i)} \exp \left(a_{j} t_{j}{ }^{(i)}\right)}{\exp \left(a_{j} t_{j}{ }^{(i)}\right)-1} \frac{\Phi_{j}\left(B^{\prime \prime \prime}(i), B_{j}^{\prime \prime}{ }_{j}^{(i)}\right)}{\Phi\left(B^{\prime \prime(i)}\right)}
\end{aligned}
$$

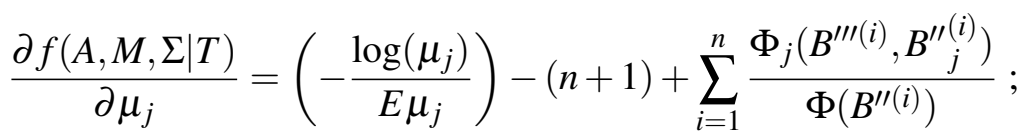

$$
\begin{aligned}
& \frac{\partial f(A, M, \Sigma \mid T)}{\partial \gamma_{j}}=-\frac{1}{2}\left(\operatorname{tr}\left(\Sigma^{-1} G^{j}\right)+p \varepsilon\left(2-\mathbf{1}^{\prime} G^{j} \mathbf{1}\right)-n \gamma_{j}\right)+ \\
& +\sum_{i=1}^{n} \frac{\frac{\phi\left(B^{\prime \prime}\right)}{2}\left[T^{\left.(i)^{\prime} \Sigma^{-1} G^{j} \Sigma^{-1} T^{(i)}-\frac{\operatorname{Adj}(\Sigma)_{j} \mathbf{1}^{\prime} G^{j} \mathbf{1}}{|\Sigma|}\right] \Phi_{j}\left(B^{\prime \prime \prime}(i), B^{\prime \prime \prime}(i)\right.}{ }_{j}\right)}{\Phi\left(B^{\prime \prime(i)}\right)} ;
\end{aligned}
$$

where $j=1, \ldots, p, B^{\prime \prime \prime}=\left\{v \in \mathbb{R}^{p-1} \mid v_{k}=B_{k}^{\prime \prime}, k=1, \ldots, p, k \neq j\right\}, \phi(x)$ is the value of the density function of p-variate normal $\operatorname{MVN}(0, \Sigma)$ in $\mathrm{x}$, and $\Phi_{j}(\cdot, y)$ is the "cumulative probability function" of a p-variate normal distribution, $\operatorname{MVN}(0, \Sigma)$, in the range $[X, \infty]$, setting $x_{j}=y$.

The initial parameter values for the optimization may be obtained by fitting a univariate Gompertz distribution for each random variable $t_{1}, t_{2}, \ldots, t_{p}$ and by computing the sample covariance matrix. In this work, the numerical integration is performed by Metropolis-Hastings Markov Chain Monte Carlo, using a multivariate normal kernel as proposal distribution for parameters $A, M$ and $\gamma$. The kernel covariance matrix is adjusted dynamically using the MCMCpack R package (Martin, 2011). All numerical routines were developed in $\mathrm{R}$ environment ( $\mathrm{R}$ Development Core Team, 2011). 


\section{Procedures for the Cumulative Normal}

Genz (1993) presents guidelines for the numerical implementations of the cumulative multivariate Normal distribution in a form that is amenable to ranges as used in this section, in particular for the implementation of $\Phi_{j}$. Making the necessary adaptations for our specific needs, we can use the following algorithm to implement $\Phi_{j}$.

Using previous notation and $l=B_{j}^{\prime \prime(i)}$ :

1. Input $\Sigma, l, j, \varepsilon, \alpha, N_{\max }$.

2. Swap j-th and last rows in $\Sigma$ and $l$ and $\mathrm{j}$-th and last columns in $\Sigma$.

3. Compute lower triangular Cholesky factor $C$ for $\Sigma$.

4. Initialize Intsum $=0, N=0$, Varsum $=0, d_{1}=\Phi\left(l_{1} / C_{1,1}\right)$ and $f_{1}=1-d_{1}$

5. Repeat

(a) Generate uniform random $w_{1}, w_{2}, \ldots, w_{k-1} \in[0,1]$

(b) For $i=2,3, \ldots, p$ set $y_{i-1}=\Phi^{-1}\left(d_{i-1}+w_{i-1}\left(1-d_{i-1}\right)\right)$,

$\left.\mathbf{I F} \mathbf{i}==\mathbf{p} d_{i}=\phi\left(\left(l_{i}-\sum_{k=1}^{i-1} C[i, k] y_{k}\right) / C[i, i]\right) / / C[i, i]\right)+1$

ELSE $d_{i}=1-\Phi\left(\left(l_{i}-\sum_{k=1}^{i-1} C[i, k] y_{k}\right) / C[i, i]\right) / C[i, i]$ $f_{i}=\left(1-d_{i}\right) f_{i-1}$.

(c) Set $N=N+1, \delta=\left(f_{k}-\right.$ Intsum $) / N$, Intsum $=$ Intsum $+\delta$, Varsum $=(N-2)$ Varsum $/ N+\delta^{2}$ and Error $=\alpha \sqrt{\text { Varsum }}$.

Until Error $<\varepsilon$ or $N=N_{\max }$.

6. Output $F=$ Intsum, Error and $N$.

\section{EXPERIMENTAL RESULTS}

This section presents some numerical experiments motivated by the Iris Flower Data Set, of the species Iris Virginica available in Anderson (2003, pp.110,111). The dataset, gives four features measured from each sample, they are the length and the width of sepal and petal, in centimeters.

We considered two hypotheses in this case study:

- H1: The features are uncorrelated, that is, the Pearson's correlation coefficient between these variables, $\rho$, is zero. The null hypothesis $H 1: \rho=0$, against $\rho \neq 0$, corresponds to the structural hypothesis

$$
h(\theta)=\left[\begin{array}{llllll}
\gamma_{5} & \gamma_{6} & \gamma_{7} & \gamma_{8} & \gamma_{9} & \gamma_{10}
\end{array}\right]^{\prime}=0
$$

- H2: The features have the following covariance structure:

$$
h(\theta)=\left[\begin{array}{lll}
\delta^{2} \gamma_{1}-\gamma_{3} & \delta^{2} \gamma_{2}-\gamma_{4} & \delta^{2} \gamma_{5}-\gamma_{6}
\end{array}\right]^{\prime}=0 .
$$


The estimated covariance matrix of the variables in the original data is

$$
\left[\begin{array}{rrrrr} 
& \text { Sepal.Length } & \text { Sepal.Width } & \text { Petal.Length } & \text { Petal.Width } \\
\text { Sepal.Length } & 0.40434286 & 0.09376327 & 0.30328980 & 0.04909388 \\
\text { Sepal.Width } & 0.09376327 & 0.10400408 & 0.07137959 & 0.04762857 \\
\text { Petal.Length } & 0.30328980 & 0.07137959 & 0.30458776 & 0.04882449 \\
\text { Petal.Width } & 0.04909388 & 0.04762857 & 0.04882449 & 0.07543265
\end{array}\right]
$$

The computed evidence value for the hypotheses $H 1$ was 0 and for $H 2$ was 0.96 on the original dataset. As expected, the results show a very strong evidence in favor of the hypothesis $H 2$ and no evidence supporting the hypothesis $H 1$.

Additionally, we performed a simulated experiment which consisted in drawing 100 samples with replacement from the original data, with the original sample size $(n=50)$. For each sample, we computed the evidences if favor of previous hypotheses. The goal was to estimate the empirical cumulative distributions for the evidences in favor of both hypotheses.

Figure 1 presents the numerical results. Each curve represents the cumulative distribution for the evidences in favor of $H 1$ (dashed line) and $H 2$ (dash-dotted line), respectively. Notice that most of the evidences for $H 1$ and $H 2$ are close to those obtained on the original data, which suggests that FBST is consistent even in the presence of a moderate sample size. Future works shall evaluate the evidence convergence for increasing sample sizes.

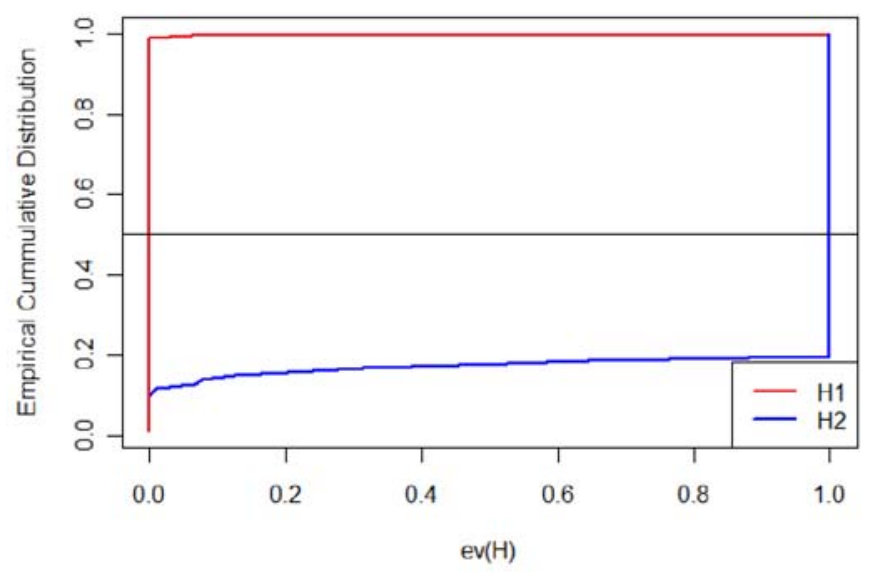

FIGURE 1. Empirical cumulative distributions for the evidence in favor of hypotheses $H 1$ (dashed) and $H 2$ (dash-dotted) computed from resamples of the original data

\section{CONCLUSION}

This paper presents several mathematical and computational details that are necessary to implement the Full Bayesian Significance Test for particular covariance structures in Gompertz type multivariate models. In studies already in progress, we hope to apply this framework for modeling medical and biological aging data. We are particularly 
interested in modeling the aging process acceleration of cancer cells by target specific chemotherapic drugs.

\section{ACKNOWLEDGMENTS}

The authors are grateful for the support of the Department of Applied Mathematics of IME-USP - the Institute of Mathematics and Statistics of the University of São Paulo, FAPESP - Fundação de Amparo à Pesquisa do Estado de São Paulo, and CNPq - Conselho Nacional de Desenvolvimento Científico e Tecnológico (grant PQ-306318-20083 ). The authors are grateful for the helpful discussions with several of their colleagues at the Bayesian research group at IME-USP, and also to Prof. Sergio Bydlowski of FMUSP - the Medicine School of the University of São Paulo, for sharing his insights and motivations concerning possible applications of our research.

\section{REFERENCES}

1. Adham, S.A. and Walker, S.G., "A multivariate Gompertz-type distribution," Journal of Applied Statistics, Vol. 28, No. 8, 2001, 1051-1065.

2. Aitchison, J. and Shen, S.M., "Logistic-Normal Distributions: Some Properties and Uses," Biometrika, 67, 1980, 261-272.

3. Anderson, T. W., "An Introduction to Multivariate Statistical Analisys,' '3rd ed, John Wiley \& Sons, New Jersey, 2003.

4. Borges,W.; Stern,J.M. The Rules of Logic Composition for the Bayesian Epistemic e-Values. Logic Journal of the IGPL, 2007, 15, 5-6, 401-420.

5. Gavrilov, L.A., Gavrilova, N.S. (1991). The Biology of Life Span: A Quantitative Approach. New York: Harwood Academic Publisher.

6. Gavrilov, L.A., Gavrilova, N.S. (2001). The Reliability Theory of Aging and Longevity. J. Theor. Biol. 213, 527-545.

7. Genz, A., "Comparison of Methods for the Computation of Multivariate Normal Probabilities," Computing Science and Statistics, 25(1), 1993, 400-405.

8. Lauretto, M., Pereira, C.A.B., Stern, J.M. and Zacks, S., "Full Bayesian significance Test Applied to Multivariate Normal Structure models," Brazilian Journal of Probability and Statistics, 17(2), 2003, 147-168.

9. Martin, A.D., Quinn, K.M., Park, J.H., “MCMCpack: Markov Chain Monte Carlo in R,” Journal of Statistical Software, 42(9), 2011, 1-21. Available at http://www.jstatsoft.org/v42/i09/

10. Pereira, C.A.B. and Stern, J.M., "Evidence and Credibility: Full Bayesian Significance Test for Precise Hypotheses," Entropy, 1, 4, 1999, 99-110.

11. Pereira, C.A.B. and Stern, J.M., "Special Characterizations of Standard Discrete Models," RevStat Statistical Journal, 6, , 2008, 199-230.

12. Pereira, C.A.B.; Wechsler, S. and Stern, J.M., "Can a Significance Test be Genuinely Bayesian?" Bayesian Analysis, 3, 1, 2008, 79-100.

13. R Development Core Team, "A Language and Environment for Statistical Computing," R Foundation for Statistical Computing, Vienna, Austria, , 2011, Available at http://www.R-project.org

14. Stern, J.M., "Cognitive Constructivism and the Epistemic Significance of Sharp Statistical Hypotheses," Tutorial book for MaxEnt 2008, The 28th International Workshop on Bayesian Inference and Maximum Entropy Methods in Science and Engineering, July 6-11 of 2008, Boracéia, São Paulo, Brazil. 
Copyright of AIP Conference Proceedings is the property of American Institute of Physics and its content may not be copied or emailed to multiple sites or posted to a listserv without the copyright holder's express written permission. However, users may print, download, or email articles for individual use. 